\title{
Hedgehog pathway as a drug target: Smoothened inhibitors in development
}

This article was published in the following Dove Press journal:

OncoTargets and Therapy

10 March 2012

Number of times this article has been viewed

\author{
Tara L Lin' \\ William Matsui ${ }^{2}$ \\ 'Division of Hematology/Oncology, \\ Department of Internal Medicine, \\ University of Kansas, Kansas City, \\ MO, USA; ${ }^{2}$ Division of Hematologic \\ Malignancies, The Sidney Kimmel \\ Comprehensive Cancer Center, Johns \\ Hopkins University School \\ of Medicine, Baltimore, MD, USA
}

\begin{abstract}
Emerging laboratory and clinical investigations demonstrate that Hedgehog signaling (Hh) represents a novel therapeutic target in various human cancers. This conserved signaling pathway precisely regulates self-renewal and terminal differentiation in embryonic development, but is typically silenced in adult tissues, with reactivation usually only during tissue repair. Aberrant Hh pathway signaling has been implicated in the pathogenesis, self-renewal, and chemotherapy resistance of a growing number of solid and hematologic malignancies. Major components of the Hh pathway include the Hh ligands (Sonic, Desert, and Indian), the transmembrane receptor Patched, the signal transducer Smoothened (Smo), and transcription factors Gli1-3 which regulate the transcription of Hh target genes. Mutations in Hh pathway genes, increased Hh signaling in tumor stroma, and Hh overexpression in self-renewing cells (cancer stem cells) have been described, and these different modes of Hh signaling have implications for the design of Hh pathway inhibitors and their integration into conventional treatment regimens. Discovery of a naturally-occurring Smo inhibitor, cyclopamine, and the identification of $\mathrm{Hh}$ pathway mutations and over expression in cancer cells prompted the development of several cyclopamine derivatives. Encouraging laboratory and in vivo data has resulted in Phase I and II clinical trials of Smo inhibitors. In this review, we will discuss the current understanding of Hh pathway signaling in malignancy and Smo antagonists in development. Recent data with these agents shows that they are well-tolerated and may be effective for subsets of patients. Challenges remain for appropriate patient selection and the optimal combination and sequence of these targeted therapies into current treatment paradigms.
\end{abstract}

Keywords: hedgehog pathway, Smoothened inhibitors, cancer stem cells

\section{Introduction}

A growing body of evidence demonstrates a role for conserved embryonic signaling pathways such as Hedgehog (Hh), Wingless, and Notch in the development of human cancers. ${ }^{1,2}$ Preclinical data in various tumor types suggests a role for Hh signaling in cancers of the skin, ${ }^{3,4}$ brain, ${ }^{5,6}$ lung, ${ }^{7}$ breast, ${ }^{8}$ prostate, ${ }^{9,10}$ colon, ${ }^{11}$ as well as hematologic malignancies including leukemia, ${ }^{12-15}$ lymphoma, ${ }^{16-19}$ and multiple myeloma (MM). ${ }^{16,20}$ Laboratory data suggests that $\mathrm{Hh}$ signaling regulates multiple pathogenic processes including tumor growth, self-renewal, and resistance to chemotherapy. Given that Hh signaling is implicated in a wide range of malignancies, there is much interest in the development of Hh pathway inhibitors as novel anticancer therapy. In this review, we will discuss the Hh pathway in cancer, mechanisms of its aberrant activation in different tumor types, and its role in the self-renewing cell population, or cancer stem cells (CSCs). We will then review published and preliminary data regarding Hh pathway inhibitors clinical trials.
Correspondence: Tara L Lin

Division of Hematology/Oncology Department of Internal Medicine, University of Kansas, 2330 Shawnee Mission Parkway, Suite 210, Mail Stop 5003, Westwood, KS 66205, USA

$\mathrm{Tel}+$ I $9 \mid 35883884$

Fax + I 9135884085

Email tlin@kumc.edu 


\section{The Hh pathway}

The Hh signaling pathway regulates cell differentiation and self-renewal in the developing embryo and is typically silenced in adult tissues. ${ }^{21}$ Aberrant Hh signaling may result from mutations in pathway genes or overexpression of signaling through other mechanisms in either tumor cells themselves or cells in the supportive tumor microenvironment. ${ }^{22-26}$ Much of our knowledge of the Hh pathway comes from studies in Drosophila, and although several major components of the pathway are well described, some details remain poorly understood. In mammals, one of three Hh pathway ligands (Desert, Indian, and Sonic) binds to the transmembrane receptor Patched (Ptch) to initiate pathway signaling. In the inactive state, Ptch exerts an inhibitory effect on the signal transducer Smoothened (Smo), and no downstream signaling occurs. When Hh ligand binds to Ptch, the inhibition on Smo is released and downstream signaling occurs, regulating the expression of the transcription factors Gli1-3 (Figure 1). Primary cilia present on most cells during interphase are involved in signal transduction, and Hh pathway components translocate during activation. In the inactive state, when $\mathrm{Hh}$ ligand is not present, Ptch is located in the primary cilia but Smo is not. When ligand binds and Ptch inhibition of Smo is released, Ptch moves out of the primary cilia and Smo moves in to facilitate interaction with Glis and associated proteins. They subsequently enter the nucleus and regulate expression of Hh target genes. ${ }^{27-32}$

Hh expression is precisely regulated through both positive and negative feedback loops which may be interrupted by mutations in Hh pathway genes themselves or epigenetic changes. Increased transcription of Hh target genes results in increased cell proliferation and survival, induction of stem cell markers, as well as promotion of bone metastases..$^{33}$ Aberrant Hh signaling has also been associated with chemotherapyresistance in gliomas, ${ }^{34}$ pancreatic cancer, ${ }^{35}$ leukemia, ${ }^{36,37}$ lymphoma, ${ }^{17,38}$ and MM. ${ }^{39}$ Interactions with other signaling pathways, including Notch, PI3K, RAS-MEK/AKT, and $\mathrm{NF}-\mathrm{\kappa B}$, to promote cancer growth, recurrence, and chemotherapy resistance have also been described. ${ }^{40-43}$

Several Smo inhibitors are in clinical development for the treatment of human cancers. Recently, emerging clinical data have demonstrated the potential activity of these agents in several diseases, particularly medulloblastoma, basal cell carcinoma (BCC), pancreatic cancer, and hematologic malignancies. Ongoing trials will evaluate the role for Smo inhibitors as single agents, as well as in combination with traditional chemotherapy. This review will discuss the mechanisms of $\mathrm{Hh}$ signaling in malignancy and the evidence for Hh signaling in CSCs. These preclinical studies provide the rationale for human trials of $\mathrm{Hh}$ inhibition in various malignancies, and we will review the progress and challenges of translating the laboratory investigations of Smo inhibitors into meaningful clinical results for patients.

\section{The Hh pathway in cancer}

Similar to its role in normal development, dysregulated $\mathrm{Hh}$ signaling results in the expression of a number of genes responsible for cell proliferation, survival, and self-renewal.
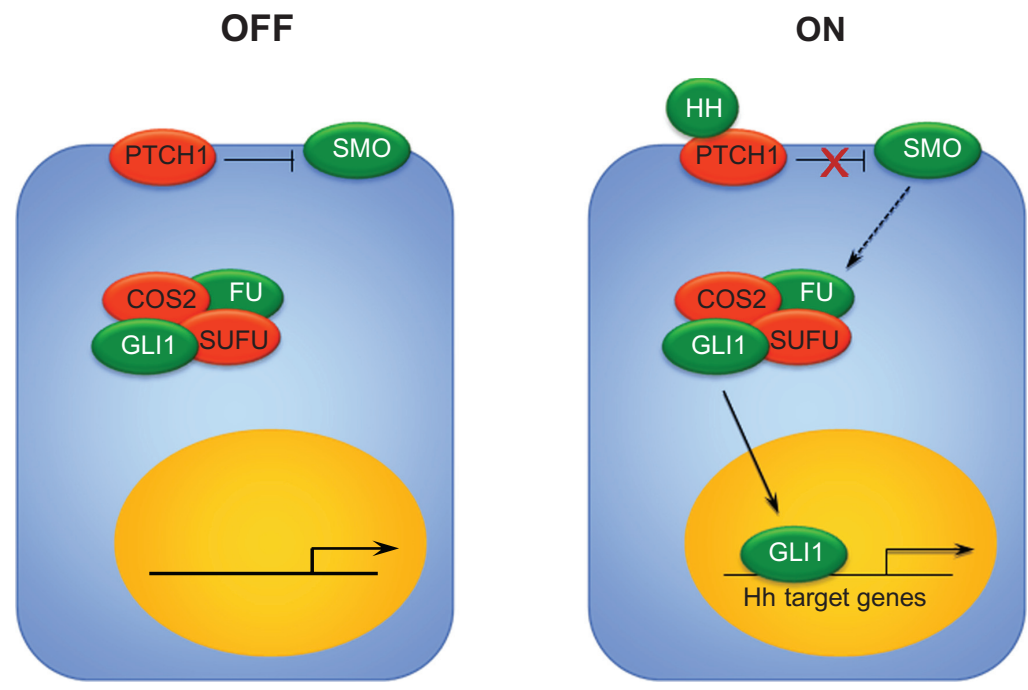

Figure I Hh signaling pathway. In the absence of Hh ligand, Ptch exerts an inhibitory effect on Smo, and no downstream signaling occurs. In the presence of Hh ligand binding to Ptch, the suppression of Smo is released. Smo interacts with Suppressor of fused (SUFU), which promotes the activation and nuclear translocation of Glil. Glil translocation results in the transcription of $\mathrm{Hh}$ target genes.

Abbreviations: Hh, Hedgehog; Ptch, Patched; Smo, Smoothened, COS, Ccoastal-2; FU, Fused. 
Aberrant Hh signaling is associated with the development of cancer, as demonstrated by the Gorlin syndrome, caused by an autosomal dominant germline mutation in the PTCH1 gene. ${ }^{4,44}$ This resultant mutated Ptch is unable to exert its tonic inhibition of Smo, resulting in hyperactivation of the pathway. Patients with Gorlin syndrome are predisposed to various malignancies, most commonly BCC and medulloblastoma. ${ }^{45}$ These observations led to the discovery of $\mathrm{Hh}$ activation in the majority of the more common sporadic form of BCC, with mutations in the PTCH1 allele occurring in up to $30 \%$ of cases $^{3}$ and $S M O$ mutations in approximately $10 \% .{ }^{46}$ In addition, mutations in Hh pathway genes have been implicated in the pathogenesis of up to $30 \%$ of sporadic medulloblastoma. ${ }^{47}$

\section{Mechanisms of Hh signaling in cancer}

Although Hh pathway gene mutations lead to inappropriate Hh signaling in $\mathrm{BCC}$ and medulloblastoma, a greater number of cancers are driven by Hh signaling through other mechanisms, either in the bulk population of cells or specifically within the CSC population. We will briefly discuss the different mechanisms of Hh signaling, and for a complete review, the reader is referred to Reference $8 .{ }^{26}$ In both BCC and medulloblastoma, Hh pathway activation results from specific gene mutations and is independent of the presence of Hh ligand binding to Ptch. This mechanism of Hh activation, which is ligand-independent and driven by specific $\mathrm{Hh}$ gene mutations within the tumor cells, is termed Type I Hh signaling (Figure 2A) ${ }^{26} \mathrm{Hh}$ inhibitors which are antagonists to Hh ligand will not be effective in overcoming this mechanism of aberrant signaling because it occurs downstream and independent of ligand due to the mutation. The other mechanisms of Hh signaling observed in cancer rely upon Hh ligand initiation of the signaling, and vary by source and recipient cells of ligand secretion.

In Type II signaling, activation of the pathway is liganddependent and autocrine, meaning it originates and is received by the tumor cells (or neighboring cells). Most data for Type II Hh signaling comes from in vitro studies in various cancers including lung, ${ }^{48,49}$ prostate,${ }^{50}$ glioblastoma, ${ }^{51,52}$ gastrointestinal, ${ }^{11,53}$ breast, ${ }^{54}$ and leukemia. ${ }^{13,15}$ These studies observed Hh expression in tumor cells and growth inhibition with Hh blockade by cyclopamine in models absent of tumor stroma. This data supports the premise that Hh ligand
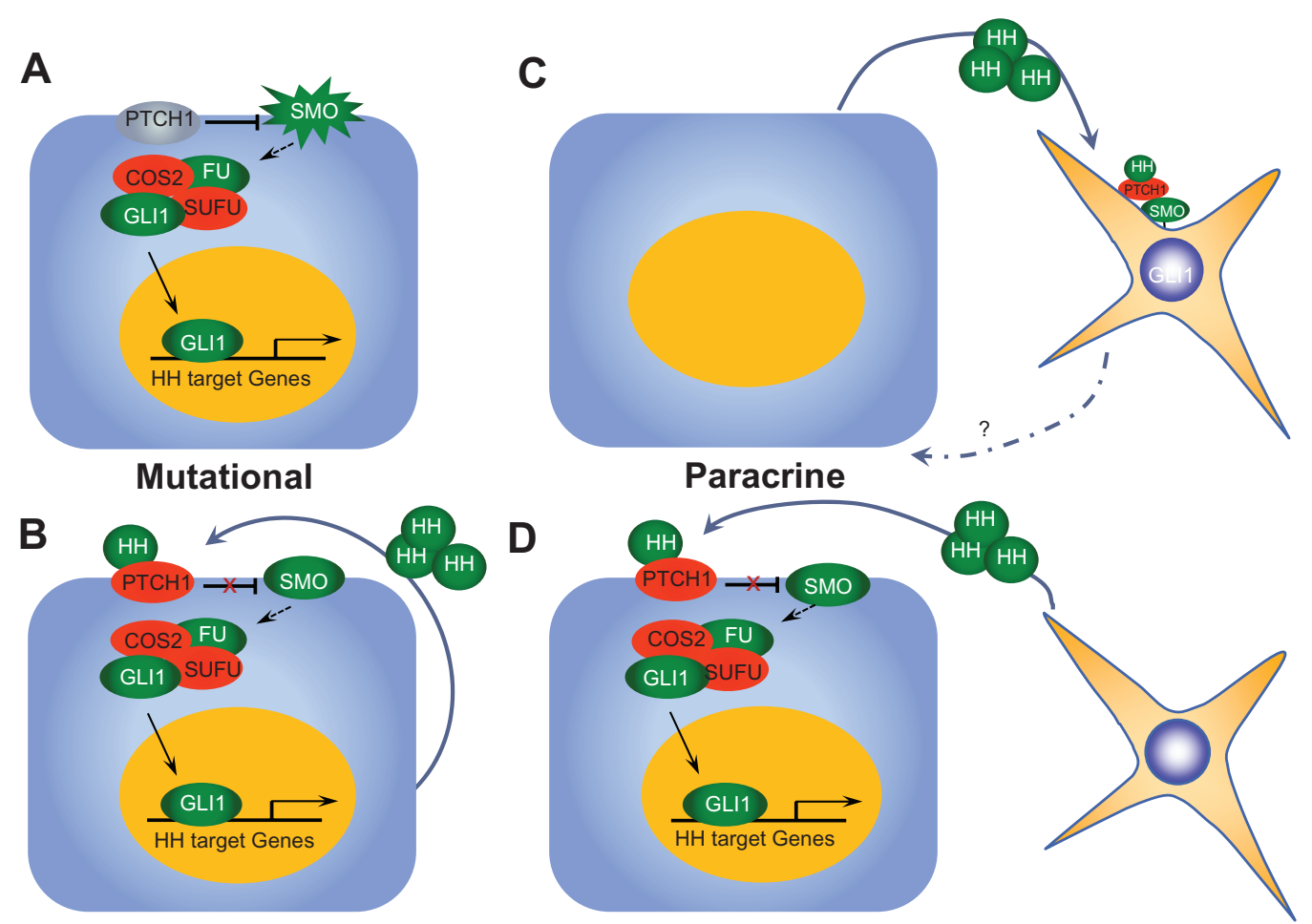

Autocrine

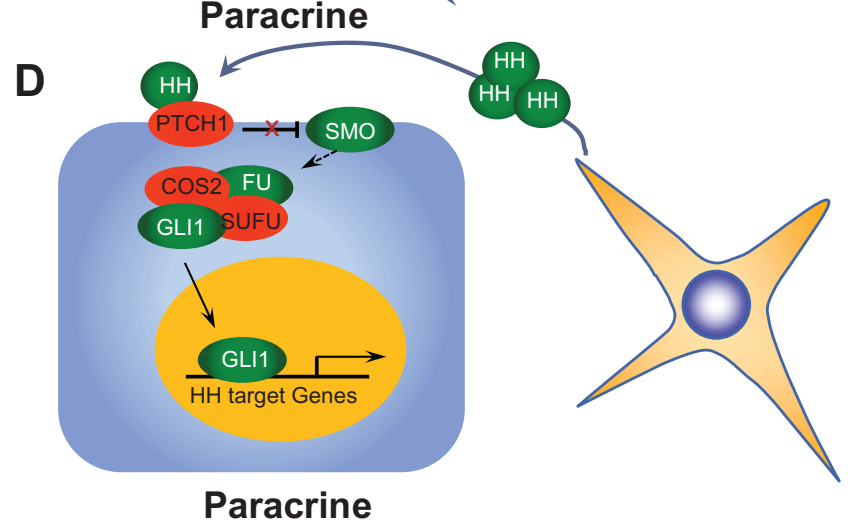

Figure 2 Modes of Hh pathway signaling. (A) Type I Hh signaling is activated by specific mutations within pathway genes within tumor cells, resulting in ligand-independent constitutive activation. (B) Type II Hh signaling results from autocrine signaling from tumor cell to tumor cell. (C) Type Illa activation results from secretion of Hh ligand by tumor cells, resulting in pathway activation in surrounding tumor stroma. (D) Type Illb Hh signaling results from Hh ligand secretion by tumor stroma, resulting in activation of the pathway within tumor cells themselves.

Abbreviation: Hh, Hedgehog. 
originates within the tumor cells and that pathway activation also occurs within tumor cells (either the same cells or neighboring cells). Several authors remain unconvinced that Type II signaling actually exists in vivo because much of this data is based on studies with higher doses of cyclopamine which exhibit some non-specific cytotoxicity. ${ }^{25,26,46,55}$ However, in our group's report of Hh signaling in acute lymphocytic leukemia (ALL), we demonstrated findings of increased $\mathrm{Hh}$ pathway expression in human ALL cell lines and clinical samples. Using a luceriferase reporter assay, we observed decreased Gli1 expression in ALL cell lines following treatment with 5E1, antagonist to Hh ligand, cyclopamine, or IPI-926 (Infinity Pharmaceuticals, Cambridge, MA), a semi-synthetic Smo inhibitor at doses which did not result in apoptosis or growth inhibition. Treatment with these $\mathrm{Hh}$ inhibitors resulted in decreased self-renewal when cells were treated alone without the presence of stromal cells both in in vitro clonogenic assays, as well as in serial transplantation models in mice. Although there is likely a contributory effect of stromally-mediated Hh signaling in ALL, we believe that our data also supports a role for autocrine, Type II Hh signaling in ALL. ${ }^{15}$ Tumors characterized by Type II signaling may be susceptible to $\mathrm{Hh}$ inhibition at either the level of $\mathrm{Hh}$ ligand binding or further downstream.

A growing body of data confirms the importance of Type III Hh signaling which is ligand dependent and paracrine; that is, ligand is secreted by one type of cell (either tumor or stroma) and Hh pathway activation occurs in another (tumor or stroma). Ligand secretion by tumor cells resulting in $\mathrm{Hh}$ signaling in supportive stromal cells is termed Type IIIa signaling, whereas ligand secretion by stromal cells resulting in Hh signaling in the tumor cells is termed reverse paracrine signaling or Type IIIb. Tumor types in which paracrine signaling has been described include prostate, ${ }^{9}$ pancreas, and metastatic colon. ${ }^{25}$ Human prostate cancer cell lines showed enhanced growth in vivo with addition of Hh ligand while no differences were seen when cells were grown alone in vitro in absence of stroma, suggesting a role for stromally mediated $\mathrm{Hh}$ signaling in promoting tumor growth. RT-PCR and in situ hybridization confirmed that increased tumor ligand expression correlated with increased mouse Gli1, Gli2, and Ptch1 from stromal cells. ${ }^{9}$ Yauch et al demonstrated similar findings of increased mouse Gli1 expression in response to human Hh ligand expression in pancreatic cancer and metastatic colon cancer in xenografts from human cell lines and primary tumors. ${ }^{25}$ Importantly, these findings from mouse models were also seen upon examination of human clinical samples comprised of tumor cells and infiltrating stromal cells in prostate, pancreatic, and metastatic colon cancer. ${ }^{9,56,57}$ Type IIIb signaling has only been described in B-cell malignancies, including leukemia, MM, and non-Hodgkin's lymphoma. ${ }^{16} \mathrm{Hh}$ ligands secreted by supportive stroma in lymph nodes, spleen, and bone marrow activated Hh signaling in tumor cells. Hh inhibition resulted in increased apoptosis associated with down regulated $\mathrm{Bcl} 2$ expression.

\section{Hh inhibition as a CSC-targeted strategy}

CSC theory states that tumors are comprised of two distinct populations of cells: a majority population of differentiated tumor cells which phenotypically characterize the disease; and a second population of rare, CSC or tumor-initiating cells, with properties of self-renewal and differentiation, responsible for disease maintenance and relapse. ${ }^{58,59} \mathrm{CSC}$ theory attempts to explain the common clinical scenario of complete response to initial chemotherapy followed by relapsed disease propagated by a small population of residual cancer cells which were undetectable following initial therapy. For many cancers, conventional chemotherapy is effective against the bulk, differentiated tumor cells. Novel strategies targeting the residual CSCs responsible for disease recurrence are needed to prolong remissions, eradicate the tumor-initiating cells, and result in long-term cure. Hh signaling has been identified as a potential CSC-specific target in various cancers. ${ }^{6,13,15,20,51,60-77}$

Techniques used to isolate and characterize CSC in vitro include aldehyde dehydrogenase expression, phenotypic markers, side population by Hoechst dye exclusion, and colony-forming assays. To date, the "gold standard" for CSC identification has been the ability of this rare population of cells to regenerate tumor consisting of both phenotypic populations, differentiated cells, and CSC in animal models. ${ }^{78} \mathrm{~A}$ detailed discussion of the in vitro and in vivo methods used to characterize CSC in various tumor types is beyond the scope of this review. The reader is referred to Reference 78 for further details of these techniques, as well to the publications cited below concerning Hh signaling and CSC. CSC theory remains controversial due to the varying techniques for identification and the discrepancies in $\mathrm{CSC}$ numbers identified in primary samples and required to recreate tumors in mice by different researchers with varying techniques. Also, the inherent heterogeneity among different tumor types as well as within specific cancer types themselves. Regardless of the exact criteria for identifying CSC or properties of "stem-ness" or whether the CSC is a primitive progenitor or a de-differentiated cell, the clinical observation holds that rare populations of cancer cells persist 
following initial therapy and cause disease recurrence, and novel strategies to target these resistant, persistent cells are needed. In fact, CSC from different cancers may share similar targets, even more so than the CSC and differentiated cell of the same cancer type. Hh signaling has been implicated in the bulk, differentiated cell populations of many human cancers, and here we review the preclinical evidence for $\mathrm{Hh}$ signaling in the CSC or self-renewing cells.

Our group demonstrated a role for Hh signaling in the self-renewing cells of MM and ALL. Flow cytometric analysis of MM cell lines following treatment with the Smo inhibitor cyclopamine resulted in decreases in the CD138negative cell fraction (the CSC population) as well as in side population (SP) cells. To assess the effects of Hh inhibition on self-renewal potential, an in vitro clonogenic assay was performed. In this assay, cells were treated with an anti-Shh antibody 5E1 to block ligand-dependent Hh signaling or the Smo inhibitor cyclopamine and then assayed for the ability to form tumor colonies in semi-solid media. Compared to a vehicle control, both 5E1 or cyclopamine significantly inhibited colony-formation by MM cell lines and primarily clinical samples. ${ }^{20}$ Furthermore, this inhibitory effect was maintained during serial replating indicative of self-renewal potential. Similarly, in ALL, treatment of human ALL cell lines with the Smo inhibitors cyclopamine or IPI-926 resulted in decreased ALDH+ cells, decreased in vitro colony-formation, and decreased serial transplantation in mice, all reflective of effects on the self-renewing cell population. ${ }^{15}$ In chronic myeloid leukemia (CML) mouse models, Smo -/- mice had longer latency to develop CML when mouse bone marrow transduced with BCR-ABL was transplanted. A decrease in the number of phenotypically primitive cells (Lin- Sca-1- c-Kit+), a surrogate for CSC, was also observed in the Smo -/- mice as compared to Smo $+/+$ mice.${ }^{67}$ Similarly, Dierks et al transduced Smo - / - fetal liver cells with BCR-ABL and observed more rapid development of CML in transplanted mice. Secondary recipients of Smo -/- cells had no development of CML at 9+ months of observation. CML stem cells were isolated by flow cytometry and treated with the Smo inhibitor cyclopamine or Hh ligand blocking antibody 5E1. Decreased colony-formation in vitro was observed with both treatments, demonstrating a role for ligand-dependent Hh signaling in mediating selfrenewal in CML. Mice transplanted with BCR-ABL infected hematopoietic stem cells were treated with cyclopamine. All untreated animals rapidly developed CML and died within 4 weeks, but cyclopamine-treated mice had a $60 \%$ survival at 7 weeks. In addition, CML from cyclopamine treated mice was unable to be serially transplanted, consistent with an observed decrease in the number of CML stem cells persisting after treatment. ${ }^{13}$

In glioblastoma multiforme, elevated Gli1 expression was seen in five of 19 primary samples and four of seven cell lines tested. Treatment with cyclopamine depleted the putative CSC population as identified by increased ALDH expression and SP. In vitro, glioblastoma multiforme neurospheres were unable to produce new neurospheres after exposure to cyclopamine, and in vivo injection of cyclopamine treated glioblastoma multiforme cells did not result in tumor formation versus untreated cells, demonstrating that Hh inhibition with cyclopamine eliminated the self-renewal capacity of these cells. ${ }^{52}$ Tanaka et al demonstrated in human breast cancer cells that the CSC population, marked by either markers CD $44^{+} \mathrm{CD} 24^{-}$ low or SP had increased Hh expression and that proliferation was limited following treatment with siRNA against Gli1. ${ }^{79}$ Similarly, Liu and colleagues isolated human breast tumor CSC by flow sorting for cells with markers CD $44^{+} \mathrm{CD} 24^{- \text {llow }}$ Lin $^{-}$. Cyclopamine or siRNA against Gli1 or Gli2 inhibited Hh signaling, resulting in diminished self-renewal capacity, mediated by $B M I-1$, a known regulator of normal stem cell self-renewal. ${ }^{65}$ Tian et al looked at the effects of $\mathrm{Hh}$ inhibition with the synthetic Smo inhibitor GDC-0449 (Genentech, South San Francisco, CA; Curis, Lexington, MA; Roche, Basel, Switzerland) in lung cancer cell lines and used SP technique to isolate and enrich for CSC. They observed expression of Smo in the SP fraction, clonogenicity restricted to the SP fraction, and that treatment with GDC-0449 reduced the number of SP cells. ${ }^{73}$ Pancreatic CSCs as characterized by ALDH expression were preferentially reduced in number as compared to differentiated tumor cells when treated with cyclopamine.${ }^{62}$ Similarly, Singh et al showed that Hh inhibition with GDC-0449 resulted in increased apoptosis of pancreatic cell line CSC as well as decreased transcription of Hh target genes. ${ }^{70}$ Other groups have described Hh signaling in the CSC of cancers of the prostate, ${ }^{60}$ stomach, ${ }^{71}$ colon, ${ }^{63}$ and ovary. ${ }^{80}$

\section{Targeting Hh signaling in cancer}

The identification of a naturally-occurring Hh pathway antagonist, cyclopamine, led to the subsequent development of synthetic and semi-synthetic derivatives of cyclopamine with increased potency and bioavailability. In addition, a commonly used anti-fungal agent, itraconazole, has also been found to have $\mathrm{Hh}$ inhibitory activity. These agents are now moving from the laboratory into clinical trials. Similar to challenges encountered in translating other targeted therapies, questions remain including the optimal way to integrate them into regimens of conventional chemotherapy. In addition, 
our growing understanding of mechanisms of Hh signaling in different malignancies raises the issue of whether there should be different approaches to using these agents which vary by the mechanism of Hh signaling in each disease. Currently, all of the Hh inhibitors in clinical development are at the level of Smo, but other agents with distinct mechanisms of action have been identified and it is possible that these may be more or less effective depending on the mode of signaling. Below, we will discuss cyclopamine, itraconazole, and Smo inhibitors in clinical trials.

\section{Cyclopamine}

In the 1960s, teratogenic effects of ingestion of the corn lily Veratrum californicum were observed, resulting in one-eyed offspring (cyclopia) in lambs. ${ }^{81}$ It was subsequently discovered that the active agent, cyclopamine, exerted its effects through Hh pathway inhibition, ${ }^{82}$ specifically acting at the level of Smo. ${ }^{83}$ Cyclopamine displayed anti-tumor activity in vitro and in vivo, but poor oral bioavailability and acid sensitivity has prevented further clinical development. One clinical report of four patients with BCC, one of whom had Gorlin syndrome, described dramatic, rapid clinical regression of the lesions in response to topical cyclopamine application versus placebo. In addition, histological and immunohistochemical analysis showed apoptosis and increased markers of differentiation in response to $\mathrm{Hh}$ inhibition. ${ }^{84}$ Although no longer in clinical development due to increased potency and bioavailability of cyclopamine derivatives, cyclopamine remains an important agent in preclinical models of Hh inhibition.

\section{Itraconazole}

Interestingly, the anti-fungal agent itraconazole was found to have Hh inhibitory properties on a drug screen of known compounds. Kim et al showed that commonly used doses of itraconazole suppressed $\mathrm{Hh}$ pathway activity and inhibited growth of medulloblastoma in vivo. It appears to act on Smo, as does cyclopamine and its synthetic derivatives, but its mechanism of Smo antagonism is distinct from that of cyclopamine and appears to limit the ciliary accumulation of Smo. ${ }^{85}$ Itraconazole is currently in studies of patients with $\mathrm{BCC}$, metastatic prostate cancer, and recurrent non-small cell lung cancer (see Table 1).

\section{Synthetic and semi-synthetic cyclopamine derivatives}

All of the $\mathrm{Hh}$ inhibitors in clinical trials currently act at the level of Smo (see Table 1 for a list of currently open clinical trials and Table 2 for a summary of findings of Smo inhibitors in clinical trials). Due to the decreased oral bioavailability and acid sensitivity of cyclopamine, semi-synthetic and synthetic derivatives have been developed. These derivatives appear to have increased potency and all are oral agents. Smo inhibitors currently under investigation appear to inhibit Smo through binding at the same portion of the transmembrane segment $6 .{ }^{86,87}$ Here, we will review the published or presented data regarding Smo inhibitors in clinical trials for cancer.

\section{Vismodegib}

Early results from patients with medulloblastoma and BCC on the initial Phase I study of GDC-0449 were published in the New England Journal of Medicine in 2009 demonstrating a role for Smo inhibitors in cancers known to be driven by Hh pathway mutations. ${ }^{88,89}$ In the first report, one patient with heavily pre-treated medulloblastoma had clinical and radiographic regression of widespread systemic metastases on vismodegib. These results were short-lived, however, with

Table I Smoothened inhibitors currently in clinical trials for cancer

\begin{tabular}{|c|c|c|c|}
\hline Drug & Sponsor & Indications & Phases \\
\hline GDC-0449 & $\begin{array}{l}\text { Genentech, Curis, } \\
\text { Roche }\end{array}$ & $\begin{array}{l}\text { Advanced solid tumors, BCC, breast, chondrosarcoma, colorectal, gastric, } \\
\text { Glioblastoma multiforme, medulloblastoma, multiple myeloma, ovarian, } \\
\text { Pancreatic, prostate sarcoma, small cell lung }\end{array}$ & Phase II \\
\hline LDE-225 & Novartis & Advanced solid tumors, BCC, chronic myeloid leukemia, pancreatic & Phase I, II \\
\hline $\begin{array}{l}\text { BMS-833923 } \\
(\mathrm{XLI} / 39)\end{array}$ & $\begin{array}{l}\text { Bristol-Myers Squibb, } \\
\text { Exelixis }\end{array}$ & $\begin{array}{l}\text { Advanced solid tumors, BCC, chronic myeloid leukemia, esophageal, } \\
\text { gastric, multiple myeloma, small cell lung }\end{array}$ & Phase I, II \\
\hline IPI-926 & Infinity & $\begin{array}{l}\text { Advanced solid tumors, chondrosarcoma, head and neck, myelofibrosis, } \\
\text { pancreatic }\end{array}$ & Phase I, II \\
\hline PF-04449913 & Pfizer & Advanced solid tumors, hematologic malignancies & Phase I \\
\hline LEQ-506 & Novartis & Advanced solid tumors & Phase I \\
\hline TAK-44I & Millenium & Advanced solid tumors & Phase I \\
\hline Itraconazole & & $\begin{array}{l}\text { BCC, metastatic prostate cancer, } \\
\text { non-small cell lung cancer }\end{array}$ & Phase II \\
\hline
\end{tabular}

Note: As listed on clinicaltrials.gov on November 9, 2011.

Abbreviation: BCC, basal cell carcinoma. 
Table 2 Summary of clinical findings from Phase I trials of Smoothened inhibitors in cancer

\begin{tabular}{|c|c|c|c|c|c|}
\hline Parameter & $\begin{array}{l}\text { GDC-0449 (vismodegib) } \\
\text { Genentech }\end{array}$ & $\begin{array}{l}\text { IPI-926 } \\
\text { Infinity }\end{array}$ & $\begin{array}{l}\text { LDE225 } \\
\text { Novartis }\end{array}$ & $\begin{array}{l}\text { BMS-833923 (XLI39) } \\
\text { BMS/Exelexis }\end{array}$ & $\begin{array}{l}\text { PF-04499 I } 3 \\
\text { Pfizer }\end{array}$ \\
\hline N (Phase I) & 68 & 104+ (ongoing) & $35+$ (ongoing) & 27 & $39+$ (ongoing) \\
\hline $\begin{array}{l}\text { Daily doses } \\
\text { explored (mg) }\end{array}$ & $150-270-540$ & $20-210 \mathrm{mg}$ & $\begin{array}{l}100-200-400- \\
800-1500\end{array}$ & $30-60-120-240-360-540$ & $\begin{array}{l}5-10-20-40-80-120- \\
180-270\end{array}$ \\
\hline GLII inhibition & Yes & & Yes & Yes & Yes \\
\hline Single dose $t_{1 / 2}$ & $>7 d$ & & $\sim 4 d(1-10) ?$ & $\sim 7 \mathrm{~d} ?$ & I7-35 hours \\
\hline MTD defined? & No (PK futility) & No & No & Yes & No \\
\hline Grade 3 toxicities & $\downarrow \mathrm{Na}$, fatigue & $\uparrow$ LFTs, fatigue & Asthenia & $\downarrow$ phos, $\uparrow$ lipase & $\begin{array}{l}\text { Hypoxia, pleural } \\
\text { effusions, } \\
\text { hemorrhagic } \\
\text { gastritis }\end{array}$ \\
\hline Most common & Muscle spasms & Nausea, fatigue & Nausea & Muscle spasms & Dysgeusia \\
\hline \multirow[t]{4}{*}{ toxicities } & Dysgeusia & & Muscle spasms & Dysgeusia & Arthralgias \\
\hline & Fatigue & & Fatigue & & Alopecia \\
\hline & & & & & Nausea \\
\hline & & & & & Vomiting \\
\hline
\end{tabular}

measurable increases in tumor size at 3 months of therapy and subsequent identification of a single amino acid substitution conferring resistance. ${ }^{89} \mathrm{~A}$ recent update of the entire Phase I cohort demonstrated that patients with $\mathrm{BCC}$ on vismodegib had a response rate of $58 \%$. Twelve of 33 advanced $\mathrm{BCC}$ subjects had been on therapy from 8.5 months to 26.5 months, with median duration of response of 12.8 months (as of January 2010, with several patients remaining on therapy and with continued response).${ }^{90}$ Responses were only observed in the patients with medulloblastoma or BCC. At the American Society of Clinical Oncology (ASCO) Annual Meeting in 2010, preliminary data was presented from eleven patients enrolled in a Phase I study of vismodegib in medulloblastoma coordinated through the Pediatric Brain Tumor Consortium. The limited data presented suggested that the drug was well-tolerated as a single agent in patients ranging from age 4-20 years. Magnetic resonance imaging scans of the knees will monitor for changes in bone development, as this was a significant side effect observed in mouse studies with $\mathrm{Hh}$ inhibition. ${ }^{91}$

More recently, encouraging Phase II results with vismodegib in 99 patients with locally advanced or metastatic BCC were presented during the European Multidisciplinary Cancer Congress in 2011. In patients with metastatic disease, disease stabilization occurred in $63 \%$ with an overall response rate of $30 \%$. In locally advanced $\mathrm{BCC}$, the overall response rate was $43 \%$ with stable disease in $40 \%$ of patients. Median duration of progression-free survival was 9.5 months. ${ }^{92}$ The plenary session at the American Association for Cancer Research Annual Meeting in 2011 featured results from 36 patients on a Phase II study of vismodegib in patients with Gorlin Syndrome/Basal Cell Nevus Syndrome. This randomized, double-blind, placebo-controlled trial was designed to evaluate the efficacy of vismodegib in preventing the development of new BCC. The data safety monitoring board discontinued the placebo arm due to statistically significant differences in the number of new $\mathrm{BCC}(0.07$ new $\mathrm{BCC} /$ month in treated patients vs 1.74 new $\mathrm{BCC} /$ month for those on placebo) and the change in size of existing BCC (decrease of $24 \mathrm{~cm}$ in vismodegib arm vs $-3 \mathrm{~cm}$ in placebo arm)..$^{93}$ The 2011 ASCO Annual Meeting featured two reports on vismodegib in combination with chemotherapy. A Phase I trial examined the combination of vismodegib with erlotinib and gemcitabine for unresectable pancreatic cancer. Observed dose-limiting toxicities of the combination included rash, nausea, infection, visual disturbances, and thrombocytopenia. Transient responses with stable disease were seen in two dose cohorts, and a dose was determined for Phase II testing. ${ }^{94}$ An NCI-sponsored, randomized, double-blind placebo-controlled trial of chemotherapy with 5-fluorouracil and oxaliplatin (FOLFOX) with or without vismodegib in previously untreated patients with advanced gastric or gastroesophageal junction cancer was reviewed at the Trials in Progress poster session at the meeting. The study plans to enroll 116 patients in order to detect a $69 \%$ increase in progression-free survival. Laboratory correlative studies to look at effects on the tumor microenvironment are also planned. ${ }^{95}$

Across these early studies, there were no dose-limiting toxicities, and common side effects were mild and included muscle spasms, dysgeusia (taste alteration), fatigue, alopecia, and nausea. Grade 3 adverse events included reversible hyponatremia, abdominal pain, fatigue, and muscle cramps. Mild to moderate side effects included dysgeusia, muscle cramps, and weight loss, with two grade 3-4 adverse events 
in the treatment arm (muscle cramps and suicide attempt). In the trial of vismodegib in patients with basal cell nevus syndrome, the drug was discontinued in $20 \%$ of patients due to adverse effects. ${ }^{92}$ Therefore, it appears that vismodegib is well tolerated.

\section{IPI-926}

IPI-926 (Infinity Pharmaceuticals, Cambridge, MA) is the only Smo inhibitor in development which is a semi-synthetic derivative of cyclopamine. Early phase clinical trial results were presented at the ASCO Annual Meeting in 2011. In the Phase I study of patients with locally advanced or metastatic solid tumors, partial responses were seen in patients with $\mathrm{BCC}$, and several patients continued on therapy for more than 1 year. ${ }^{96}$ Preliminary findings from a Phase $\mathrm{Ib} / \mathrm{II}$ study of IPI-926 in combination with gemcitabine in patients with untreated metastatic pancreatic cancer were also presented. Median progression free survival was approximately 5.5 months with five of 16 patients demonstrating a partial response to the combination. ${ }^{97}$ In both studies, the drug was well-tolerated with mild effects of nausea, fatigue, muscle spasms, and dysgeusia observed. There was also asymptomatic and reversible elevation of liver function tests.

\section{LDE225}

Two recent reports comment on the clinical experience with LDE225 (Novartis, Basel, Switzerland). Preliminary findings from a Phase I dose escalation study of LDE225 were reported at the ASCO Annual Meeting in 2010. One patient with medulloblastoma maintained a partial response for 4 months and five additional patients with various malignancies including lung cancer and BCC have tolerated therapy for more than 4 months. ${ }^{98}$ A topical formulation of LDE225 was also recently tested in patients with Gorlin syndrome and BCC. In this double-blind, randomized, vehicle-controlled intraindividual study, $27 \mathrm{BCC}$ in eight patients were treated with LDE225 cream or vehicle twice daily for 4 weeks. In twelve of $13 \mathrm{BCC}$, there were three complete responses and nine partial responses compared to only one partial response in 14 vehicle treated BCC. ${ }^{99}$ Similar to other Smo inhibitors, the oral drug was well-tolerated with side effects of fatigue, nausea, muscle cramps, and dysgeusia being most common. The topical formulation was well-tolerated with no skin irritation.

\section{BMS-833923 (XLI 39)}

BMS-833923 (XL139) (Bristol Myers Squibb, New York, NY; Exelixis, South San Francisco, CA) is also being studied in early phase trials as a single agent in advanced and metastatic solid tumors. There are ongoing clinical trials looking at this drug in combination with standard chemotherapy in MM, gastric and esophageal cancers, and small cell lung cancer. Data was presented at the American Association for Cancer Research-National Cancer Institute-European Organization for Research and Treatment of Cancer Annual Meeting in 2009. One patient with medulloblastoma continues on study for more than 10 months with stable disease. A second subject with Gorlin syndrome had a partial response to therapy which was ongoing at the time of the report. ${ }^{100}$ At the 2011 American Society of Hematology meeting, Huff et al presented Phase I data on BMS-833293 in patients with MM. BMS-833923 was given either as monotherapy, or in combination with lenalidomide plus dexamethasone, or with bortezomib in patients with relapsed or refractory MM. No clinical response data had been analyzed to date. ${ }^{101}$ In both trials, the drug was well-tolerated with the most common side effects being dysgeusia, muscle spasms, and nausea. There was one episode of grade 2 lipase elevation and pancreatitis.

\section{PF-044499I 3}

Preliminary data was presented in abstract form with PF-04449913 (Pfizer, Manhattan, NY) at the 2011 American Society of Hematology Annual Meeting. The drug was administered as a single agent in escalating doses in a Phase Ia study of 32 patients with various hematologic malignancies including acute myeloid leukemia, CML in lymphoid blast crisis, myelodysplastic syndrome, myelofibrosis, and chronic myelomonocytic leukemia. Some evidence of response was seen in all disease subsets, and one patient with acute myeloid leukemia evolved from chronic myelomonocytic leukemia achieved a complete remission with incomplete blood count recovery. Five patients with acute myeloid leukemia had significant reduction in circulating blast counts, and one patient with T315I mutation CML in lymphoid blast crisis achieved a major cytogenetic response with loss of the T315I mutation. Other patients had hematologic improvement or disease stabilization. The drug was relatively well-tolerated with the most common side effects being dysgeusia, alopecia, nausea, and arthralgias. ${ }^{102}$ An expanded multicenter Phase $\mathrm{Ib} / \mathrm{II}$ study is planned in combination with chemotherapy in hematologic malignancies as well.

\section{Discussion}

Preclinical in vitro and in vivo data with Hh signaling demonstrate a role for the pathway in the pathogenesis, self-renewal, and chemotherapy resistance in a variety of human cancers. Years of laboratory investigations have led to Phase I trials 
of several Smo inhibitors which appear to be relatively welltolerated either as single agents or in combination regimens with conventional chemotherapy. Modest side effects include nausea, dysgeusia, muscle cramps, and fatigue, with rare grade 3 adverse events observed. It is unclear, however, what side effects may occur in children, as permanent defects in bone growth have been seen in mouse models of Hh inhibitors $^{91}$ which may complicate their clinical development for pediatric tumors such as medulloblastoma. The known teratogenic effects of cyclopamine caution for the need to prevent pregnancy in treated subjects. Encouraging preliminary results have led to ongoing clinical trials in adults in hematologic malignancies, pancreatic cancer, glioblastoma, gastrointestinal tumors, lung cancer, and other advanced solid tumors. These early trials may suggest how to incorporate Smo inhibitors into treatment regimens with conventional chemotherapy. Interestingly, many of these trials incorporate prolonged post-chemotherapy maintenance therapy with the Smo inhibitor, reflecting an understanding of the potential role of Hh signaling in maintaining the CSC population which persists after initial therapy.

Despite early enthusiasm and preclinical successes, challenges remain in the development of Smo inhibitors. Drug resistance is an important concern. Preclinical models suggest several potential mechanisms of resistance, but the most compelling data comes from one clinical example, the first patient treated with the Smo inhibitor vismodegib for refractory medulloblastoma. Despite an initial dramatic response to therapy, resistant disease emerged after 3 months with relapse at multiple sites. ${ }^{89}$ Tumor biopsies taken both before and after vismodegib provided important insights into the mechanisms of resistance to Smo inhibitors. Yauch and colleagues were able to identify a single amino acid substitution in a conserved aspartate acid residue of Smo. This mutation retained Smo activity but interfered with vismodegib binding, preventing the drug effect. Whether this mutation arose in the setting of vismodegib therapy or was present at levels too low to be detected pre-treatment remains unclear. In mouse models of medulloblastoma, the same mutation was identified in a tumor resistant to vismodegib as well. ${ }^{103}$ Similar to the development of BCR-ABL tyrosine kinase inhibitors which retain activity against mutations conferring resistance to imatinib, studies are underway to develop second-generation Smo inhibitors which remain effective in the face of known Smo mutations conferring drug resistance. ${ }^{104}$ Other mechanisms of resistance that have been identified in preclinical models include amplification of $\mathrm{Hh}$ signaling molecules downstream of Smo (cf, Gli2), ${ }^{104}$ amplification of Hh target genes, ${ }^{105}$ and upregulation of signaling pathways which interact with $\mathrm{Hh}$, such as PI3K. ${ }^{41}$ In addition, aberrant Hh signaling may result from pathway activation downstream of Smo. Hh inhibitors which act at the level of the Gli transcription factors are also under investigation in the laboratory, ${ }^{106}$ and may prove effective in the setting of Smo inhibitor resistance or in combination. For example, arsenic trioxide, used in the treatment of acute promyelocytic leukemia, has been shown to inhibit Gli proteins. ${ }^{107,108}$

Preclinical testing of Smo inhibitors has identified tumor types which appear dependent on Hh signaling for their growth, self-renewal, and chemotherapy resistance. Hh signaling does not appear consistent throughout any tumor type, including those with specific activating mutations such as BCC and medulloblastoma, in which a majority of tumors may be dependent on the pathway. At this time, testing to know which particular patients and tumors are dependent on Hh signaling is not available. Efficacy results from early phase trials of these agents should be interpreted cautiously, as the effects may be more pronounced in only specific subsets of patients, and we are currently unable to prospectively identify them. The potential to profile an individual's own tumor for dependence on Hh or other signaling pathways would better direct patients into clinical trials and suggest potential synergistic combinations of inhibitors. However, current techniques do not permit this level of personalized medicine.

The development of Smo inhibitors represents an exciting advance in cancer therapy. Hh signaling is a cancer-specific target in adults, and likely a CSC-specific target in several diseases, with the potential to have efficacy as single agents and in order to sensitize tumors to chemotherapy. As these clinical trials provide tumor samples and outcomes suggesting which patients may benefit, additional questions will need to be answered through laboratory correlative studies to better identify which patients and tumor types are most likely to be responsive to these interventions. Clinical trials of CSC-targeted therapies such as Hh inhibition may not produce early measurable clinical responses, but may instead prolong survival as the CSC are extinguished over time. In cancers where the role of Hh signaling may be due to a tumor-stromal interaction, Hh inhibition alone may result in a cytostatic effect with no measurable benefit radiographically but an improvement in survival. These factors must be considered as clinical trials incorporate Smo inhibitors with traditional chemotherapy, and overall survival represents the ideal endpoint to assess efficacy of a treatment such as this with potential CSC- or stroma-specific effects. A better 
understanding of the role of Hh signaling in the response of tumors to traditional therapy, as well as the interactions of the Hh pathway with other signaling pathways, may suggest combinations of Smo inhibitors with other pathway inhibitors for further study.

\section{Disclosure}

The authors report no conflicts of interest in this work.

\section{References}

1. Beachy PA, Karhadkar SS, Berman DM. Tissue repair and stem cell renewal in carcinogenesis. Nature. 2004;432(7015):324-331.

2. Taipale J, Beachy P. The Hedgehog and wnt signalling pathways in cancer. Nature. 2001;411(6835):349-354.

3. Gailani MR, Ståhle-Bäckdahl M, Leffell DJ, et al. The role of the human homologue of Drosophila patched in sporadic basal cell carcinomas. Nat Genet. 1996;14(1):78-81.

4. Hahn $\mathrm{H}$, Wicking $\mathrm{C}$, Zaphiropoulous $\mathrm{PG}$, et al. Mutations of the human homolog of Drosophila patched in the nevoid basal cell carcinoma syndrome. Cell. 1996;85(6):841-851.

5. Zurawel RH, Allen C, Chiappa S, et al. Analysis of PTCH/SMO/SHH pathway genes in medulloblastoma. Genes Chromosomes Cancer. 2000;27(1):44-51.

6. Bar EE, Chaudhry A, Lin A, et al. Cyclopamine-mediated hedgehog pathway inhibition depletes stem-like cancer cells in glioblastoma. Stem Cells. 2007;25(10):2524-2533.

7. Watkins DN, Berman DM, Baylin SB. Hedgehog signaling: progenitor phenotype in small-cell lung cancer. Cell Cycle. 2003;2(3):196-198.

8. Vorechovský I, Benediktsson KP, Toftgård R. The patched/hedgehog/ Smoothened signalling pathway in human breast cancer: no evidence for H133Y SHH, PTCH and SMO mutations. Eur J Cancer. 1999;35(5):711-713.

9. Fan L, Pepicelli C, Dibble C, et al. Hedgehog signaling promotes prostate xenograft tumor growth. Endocrinology. 2004;145(8):3961-3970.

10. Karhadkar SS, Bova GS, Abdallah N, et al. Hedgehog signalling in prostate regeneration, neoplasia and metastasis. Nature. 2004; 431(7009): 707-712.

11. Berman D, Karhadkar S, Maitra A, et al. Widespread requirement for Hedgehog ligand stimulation in growth of digestive tract tumours. Nature. 2003;425(6960):846-851

12. Bai L, Chiu C, Lin C, et al. Differential expression of Sonic hedgehog and Gli1 in hematological malignancies. Leukemia. 2008;22(1):226-228.

13. Dierks C, Beigi R, Guo G, et al. Expansion of Bcr-Abl-positive leukemic stem cells is dependent on Hedgehog pathway activation. Cancer Cell. 2008;14(3):238-249.

14. Warzecha J, Bonke L, Koehl U, et al. The hedgehog inhibitor cyclopamine induces apoptosis in leukemic cells in vitro. Leuk Lymphoma. 2008;49(12):2383-2386.

15. Lin TL, Wang QH, Brown P, et al. Self-renewal of acute lymphocytic leukemia cells is limited by the Hedgehog pathway inhibitors cyclopamine and IPI-926. PLoS One. 2010;5(12):e15262.

16. Dierks C, Grbic J, Zirlik K, et al. Essential role of stromally induced hedgehog signaling in B-cell malignancies. Nat Med. 2007; 13(8):944-951.

17. Hegde G, Munger C, Emanuel K, et al. Targeting of sonic hedgehogGLI signaling: a potential strategy to improve therapy for mantle cell lymphoma. Mol Cancer Ther. 2008;7(6):1450-1460.

18. Kawahara T, Kawaguchi-Ihara N, Okuhashi Y, Itoh M, Nara N, Tohda S. Cyclopamine and quercetin suppress the growth of leukemia and lymphoma cells. Anticancer Res. 2009;29(11):4629-4632.

19. Singh R, Kim J, Davuluri Y, et al. Hedgehog signaling pathway is activated in diffuse large B-cell lymphoma and contributes to tumor cell survival and proliferation. Leukemia. 2010;24(5):1025-1036.
20. Peacock CD, Wang Q, Gesell GS, et al. Hedgehog signaling maintains a tumor stem cell compartment in multiple myeloma. Proc Natl Acad Sci U S A. 2007;104(10):4048-4053.

21. Ingham $\mathrm{P}, \mathrm{McMahon} \mathrm{A}$. Hedgehog signaling in animal development: paradigms and principles. Genes Dev. 2001;15(23):3059-3087.

22. Wicking C, McGlinn E. The role of hedgehog signalling in tumorigenesis. Cancer Lett. 2001;173(1):1-7.

23. Ruiz i Altaba A, Sánchez P, Dahmane N. Gli and hedgehog in cancer: tumours, embryos and stem cells. Nat Rev Cancer. 2002; 2(5):361-372.

24. Ruiz i Altaba A. Therapeutic inhibition of Hedgehog-GLI signaling in cancer: epithelial, stromal, or stem cell targets? Cancer Cell. 2008;14(4):281-283.

25. Yauch R, Gould S, Scales S, et al. A paracrine requirement for hedgehog signalling in cancer. Nature. 2008;455(7211):406-410.

26. Scales S, de Sauvage F. Mechanisms of Hedgehog pathway activation in cancer and implications for therapy. Trends Pharmacol Sci. 2009;30(6):303-312.

27. Corbit KC, Aanstad P, Singla V, Norman AR, Stainier DY, Reiter JF. Vertebrate Smoothened functions at the primary cilium. Nature. 2005;437(7061):1018-1021.

28. Haycraft CJ, Banizs B, Aydin-Son Y, Zhang Q, Michaud EJ, Yoder BK. Gli2 and Gli3 localize to cilia and require the intraflagellar transport protein polaris for processing and function. PLoS Genet. 2005;1(4):e53.

29. Rohatgi R, Milenkovic L, Scott MP. Patched1 regulates hedgehog signaling at the primary cilium. Science. 2007;317(5836):372-376.

30. Kim J, Kato M, Beachy PA. Gli2 trafficking links Hedgehogdependent activation of Smoothened in the primary cilium to transcriptional activation in the nucleus. Proc Natl Acad Sci U S A. 2009;106(51):21666-21671.

31. Wilson CW, Chen MH, Chuang PT. Smoothened adopts multiple active and inactive conformations capable of trafficking to the primary cilium. PLoS One. 2009;4(4):e5182.

32. Rubin LL, de Sauvage FJ. Targeting the Hedgehog pathway in cancer. Nat Rev Drug Discov. 2006;5(12):1026-1033.

33. Katoh Y, Katoh M. Hedgehog target genes: mechanisms of carcinogenesis induced by aberrant hedgehog signaling activation. Curr Mol Med. 2009;9(7):873-886.

34. Cui D, Xu Q, Wang K, Che X. Gli1 is a potential target for alleviating multidrug resistance of gliomas. J Neurol Sci. 2010;288(1-2):156-166.

35. Olive KP, Jacobetz MA, Davidson CJ, et al. Inhibition of Hedgehog signaling enhances delivery of chemotherapy in a mouse model of pancreatic cancer. Science. 2009;324(5933):1457-1461.

36. Kobune M, Takimoto R, Murase K, et al. Drug resistance is dramatically restored by hedgehog inhibitors in CD34+ leukemic cells. Cancer Sci. 2009;100(5):948-955.

37. Queiroz KC, Ruela-de-Sousa RR, Fuhler GM, et al. Hedgehog signaling maintains chemoresistance in myeloid leukemic cells. Oncogene. 2010;29(48):6314-6322.

38. Singh RR, Kunkalla K, Qu C, et al. ABCG2 is a direct transcriptional target of hedgehog signaling and involved in stroma-induced drug tolerance in diffuse large B-cell lymphoma. Oncogene. 2011;30(49):4874-4886.

39. Matsui W, Wang Q, Barber J, et al. Clonogenic multiple myeloma progenitors, stem cell properties, and drug resistance. Cancer Res. 2008;68(1):190-197.

40. Schreck KC, Taylor P, Marchionni L, et al. The Notch target Hes1 directly modulates Glil expression and Hedgehog signaling: a potential mechanism of therapeutic resistance. Clin Cancer Res. 2010;16(24):6060-6070.

41. Buonamici S, Williams J, Morrissey M, et al. Interfering with resistance to Smoothened antagonists by inhibition of the PI3K pathway in medulloblastoma. Sci Transl Med. 2010;2(51):51ra70.

42. Kasperczyk H, Baumann B, Debatin KM, Fulda S. Characterization of sonic hedgehog as a novel NF-kappaB target gene that promotes NF-kappaB-mediated apoptosis resistance and tumor growth in vivo. FASEB J. 2009;23(1):21-33. 
43. Stecca B, Mas C, Clement V, et al. Melanomas require HEDGEHOGGLI signaling regulated by interactions between GLI1 and the RASMEK/AKT pathways. Proc Natl Acad Sci U S A. 2007;104(14): 5895-5900.

44. Johnson R, Rothman A, Xie J, et al. Human homolog of patched, a candidate gene for the basal cell nevus syndrome. Science. 1996; 272(5268):1668-1671.

45. Gorlin RJ. Nevoid basal-cell carcinoma syndrome. Medicine (Baltimore). 1987;66(2):98-113.

46. Xie J, Murone M, Luoh SM, et al. Activating Smoothened mutations in sporadic basal-cell carcinoma. Nature. 1998;391(6662):90-92.

47. Thompson MC, Fuller C, Hogg TL, et al. Genomics identifies medulloblastoma subgroups that are enriched for specific genetic alterations. J Clin Oncol. 2006;24(12):1924-1931.

48. Watkins D, Berman D, Burkholder S, Wang B, Beachy P, Baylin S. Hedgehog signalling within airway epithelial progenitors and in smallcell lung cancer. Nature. 2003;422(6929):313-317.

49. Yuan Z, Goetz JA, Singh S, et al. Frequent requirement of hedgehog signaling in non-small cell lung carcinoma. Oncogene. 2007; 26(7):1046-1055.

50. Karhadkar S, Bova G, Abdallah N, et al. Hedgehog signalling in prostate regeneration, neoplasia and metastasis. Nature. 2004; 431(7009):707-712.

51. Clement V, Sanchez P, de Tribolet N, Radovanovic I, Ruiz i Altaba A HEDGEHOG-GLI1 signaling regulates human glioma growth, cancer stem cell self-renewal, and tumorigenicity. Curr Biol. 2007; 17(2):165-172.

52. Bar E, Chaudhry A, Lin A, et al. Cyclopamine-mediated hedgehog pathway inhibition depletes stem-like cancer cells in glioblastoma. Stem Cells. 2007;25(10):2524-2533.

53. Varnat F, Duquet A, Malerba M, et al. Human colon cancer epithelial cells harbour active HEDGEHOG-GLI signalling that is essential for tumour growth, recurrence, metastasis and stem cell survival and expansion. EMBO Mol Med. 2009;1(6-7):338-351.

54. Mukherjee S, Frolova N, Sadlonova A, et al. Hedgehog signaling and response to cyclopamine differ in epithelial and stromal cells in benign breast and breast cancer. Cancer Biol Ther. 2006;5(6):674-683.

55. Zhang J, Lipinski R, Shaw A, Gipp J, Bushman W. Lack of demonstrable autocrine hedgehog signaling in human prostate cancer cell lines. J Urol. 2007;177(3):1179-1185.

56. Tian H, Callahan C, DuPree K, et al. Hedgehog signaling is restricted to the stromal compartment during pancreatic carcinogenesis. Proc Natl Acad Sci U S A. 2009;106(11):4254-4259.

57. Bailey JM, Mohr AM, Hollingsworth MA. Sonic hedgehog paracrine signaling regulates metastasis and lymphangiogenesis in pancreatic cancer. Oncogene. 2009;28(40):3513-3525.

58. Reya T, Morrison S, Clarke M, Weissman I. Stem cells, cancer, and cancer stem cells. Nature. 2001;414(6859):105-111.

59. O'Brien CA, Kreso A, Jamieson CH. Cancer stem cells and self-renewal. Clin Cancer Res. 2010;16(12):3113-3120.

60. Chang HH, Chen BY, Wu CY, et al. Hedgehog overexpression leads to the formation of prostate cancer stem cells with metastatic property irrespective of androgen receptor expression in the mouse model J Biomed Sci. 2011;18:6.

61. Enguita-Germán M, Schiapparelli P, Rey JA, Castresana JS. CD133+ cells from medulloblastoma and PNET cell lines are more resistant to cyclopamine inhibition of the sonic hedgehog signaling pathway than CD133-cells. Tumour Biol. 2010;31(5):381-390.

62. Feldmann G, Dhara S, Fendrich V, et al. Blockade of hedgehog signaling inhibits pancreatic cancer invasion and metastases: a new paradigm for combination therapy in solid cancers. Cancer Res. 2007;67(5):2187-2196.

63. Gulino A, Ferretti E, De Smaele E. Hedgehog signalling in colon cancer and stem cells. EMBO Mol Med. 2009;1(6-7):300-302.

64. Kawaguchi-Ihara N, Okuhashi Y, Itoh M, Murohashi I, Nara N, Tohda S. Promotion of the self-renewal capacity of human leukemia cells by sonic hedgehog protein. Anticancer Res. 2011;31(3):781-784.
65. Liu S, Dontu G, Mantle ID, et al. Hedgehog signaling and Bmi-1 regulate self-renewal of normal and malignant human mammary stem cells. Cancer Res. 2006;66(12):6063-6071.

66. Mao L, Xia YP, Zhou YN, et al. A critical role of Sonic Hedgehog signaling in maintaining the tumorigenicity of neuroblastoma cells. Cancer Sci. 2009;100(10):1848-1855.

67. Zhao C, Chen A, Jamieson CH, et al. Hedgehog signalling is essential for maintenance of cancer stem cells in myeloid leukaemia. Nature. 2009;458(7239):776-779.

68. Zhang C, Li C, He F, Cai Y, Yang H. Identification of CD44+CD24+ gastric cancer stem cells. J Cancer Res Clin Oncol. 2011;137(11): 1679-1686.

69. Schiapparelli P, Shahi MH, Enguita-Germán M, et al. Inhibition of the sonic hedgehog pathway by cyplopamine reduces the CD133+/ CD15+ cell compartment and the in vitro tumorigenic capability of neuroblastoma cells. Cancer Lett. 2011;310(2):222-231.

70. Singh BN, Fu J, Srivastava RK, Shankar S. Hedgehog signaling antagonist GDC-0449 (Vismodegib) inhibits pancreatic cancer stem cell characteristics: molecular mechanisms. PLoS One. 2011; 6(11):e27306.

71. Song Z, Yue W, Wei B, et al. Sonic hedgehog pathway is essential for maintenance of cancer stem-like cells in human gastric cancer. PLoS One. 2011;6(3):e17687.

72. Takebe N, Warren RQ, Ivy SP. Breast cancer growth and metastasis: interplay between cancer stem cells, embryonic signaling pathways and epithelial-to-mesenchymal transition. Breast Cancer Res. 2011;13(3):211.

73. Tian F, Mysliwietz J, Ellwart J, Gamarra F, Huber RM, Bergner A. Effects of the Hedgehog pathway inhibitor GDC-0449 on lung cancer cell lines are mediated by side populations. Clin Exp Med. 2011 Apr 26. [Epub ahead of print].

74. Tang SN, Fu J, Nall D, Rodova M, Shankar S, Srivastava RK. Inhibition of sonic hedgehog pathway and pluripotency maintaining factors regulate human pancreatic cancer stem cell characteristics. Int J Cancer. 2011 Jul 27. [Epub ahead of print].

75. Uchida H, Arita K, Yunoue S, et al. Role of sonic hedgehog signaling in migration of cell lines established from CD133-positive malignant glioma cells. J Neurooncol. 2011;104(3):697-704.

76. Wang X, Venugopal C, Manoranjan B, et al. Sonic hedgehog regulates Bmil in human medulloblastoma brain tumor-initiating cells. Oncogene. 2012;31(2):187-199.

77. Watkins DN, Berman DM, Burkholder SG, Wang B, Beachy PA, Baylin SB. Hedgehog signalling within airway epithelial progenitors and in small-cell lung cancer. Nature. 2003;422(6929): 313-317.

78. Clarke MF, Dick JE, Dirks PB, et al. Cancer stem cells - perspectives on current status and future directions: AACR Workshop on cancer stem cells. Cancer Res. 2006;66(19):9339-9344.

79. Tanaka H, Nakamura M, Kameda C, et al. The Hedgehog signaling pathway plays an essential role in maintaining the CD44+CD24-/low subpopulation and the side population of breast cancer cells. Anticancer Res. 2009;29(6):2147-2157.

80. Ray A, Meng E, Reed E, Shevde LA, Rocconi RP. Hedgehog signaling pathway regulates the growth of ovarian cancer spheroid forming cells Int J Oncol. 2011;39(4):797-804

81. Binns W, James LF, Shupe JL, Everett G. A congenital cyclopian-type malformation in lambs induced by maternal ingestion of a range plant, veratrum californicum. Am J Vet Res. 1963;24:1164-1175.

82. Cooper MK, Porter JA, Young KE, Beachy PA. Teratogen-mediated inhibition of target tissue response to Shh signaling. Science. 1998;280(5369):1603-1607.

83. Chen JK, Taipale J, Cooper MK, Beachy PA. Inhibition of Hedgehog signaling by direct binding of cyclopamine to Smoothened. Genes Dev. 2002;16(21):2743-2748.

84. Tabs S, Avci O. Induction of the differentiation and apoptosis of tumor cells in vivo with efficiency and selectivity. Eur J Dermatol. 2004;14(2):96-102. 
85. Kim J, Tang JY, Gong R, et al. Itraconazole, a commonly used antifungal that inhibits Hedgehog pathway activity and cancer growth. Cancer Cell. 2010;17(4):388-399.

86. Chen JK, Taipale J, Young KE, Maiti T, Beachy PA. Small molecule modulation of Smoothened activity. Proc Natl Acad Sci USA. 2002;99(22):14071-14076.

87. Rominger CM, Bee WL, Copeland RA, et al. Evidence for allosteric interactions of antagonist binding to the smoothened receptor. J Pharmacol Exp Ther. 2009;329(3):995-1005.

88. Von Hoff DD, LoRusso PM, Rudin CM, et al. Inhibition of the hedgehog pathway in advanced basal-cell carcinoma. $N$ Engl J Med. 2009;361(12):1164-1172.

89. Rudin CM, Hann CL, Laterra J, et al. Treatment of medulloblastoma with hedgehog pathway inhibitor GDC-0449. N Engl J Med. 2009;361(12):1173-1178.

90. Lorusso PM, Jimeno A, Dy G, et al. Pharmacokinetic dose-scheduling study of hedgehog pathway inhibitor vismodegib (GDC-0449) in patients with locally advanced or metastatic solid tumors. Clin Cancer Res. 2011;17(17):5774-5782.

91. Kimura H, Ng JM, Curran T. Transient inhibition of the Hedgehog pathway in young mice causes permanent defects in bone structure. Cancer Cell. 2008;13(3):249-260.

92. Dirix L, Migden M, Oro A. A pivotal multicenter trial evaluating efficacy and safety of the Hedgehog pathway inhibitor (HPI) vismodegib in patients with advanced basal cell carcinoma (BCC) [abstract]. 2011 European Multidisciplinary Cancer Congress; September 23-24, 2011; Stockholm, Sweden; 2011:1BA.

93. Tang J, Mackay-Wiggan J, Aszterbaum M. An investigator-initiated, phase II randomized, double-blind, placebo-controlled trial of GDC0449 for prevention of BCCs in basal cell nevus syndrome (BCNS) patients (abstract). American Association for Cancer Research 102 nd Annual Meeting; April 2-6, 2011; Orlando, FL; 2011:LB-1.

94. Palmer S, Erlichman C, Fernandez-Zapico M. Phase I trial erlotinib, gemcitabine, and the hedgehog inhibitor, GDC-0449 [abstract]. J Clin Oncol. 2011;29:3092.

95. Cohen D, Liebes L, Xu R. A randomized, double-blind placebo-controlled phase II study of FOLFOX with or without GDC-0449 (vismodegib) in patients with advanced gastric or gastroesophageal junction carcinoma (NCI 8376) [abstract]. J Clin Oncol. 2011;29:TPS173.

96. Rudin C, Jimeno A, Miller W. A phase I study of IPI-926, a novel hedgehog pathway inhibitor, in patients with advanced or metastatic solid tumors [abstract]. J Clin Oncol. 2011;29:3014.
97. Stephenson J, Richards D, Wolpin B. The safety of IPI-926, a novel hedgehog pathway inhibitor, in combination with gemcitabine in patients with metastatic pancreatic cancer [abstract]. J Clin Oncol. 2011;29:4114.

98. Rodon Ahnert J, Baselga J, Tawbi H, et al. A phase I dose-escalation study of LDE225, a smoothened (Smo) antagonist, in patients with advanced solid tumors [abstract]. J Clin Oncol. 2010;28:15s:2500.

99. Skvara H, Kalthoff F, Meingassner JG, et al. Topical treatment of Basal cell carcinomas in nevoid Basal cell carcinoma syndrome with a smoothened inhibitor. J Invest Dermatol. 2011;131(8):1735-1744.

100. Siu L, Papadapoulos K, Alberts S. A first-in-human phase 1study of an oral hedgehog pathway antagonist, BMS-833923 (XL139), in subjects with advanced or metastatic solid tumors. AACR-NCI-EORTC International Conference on Molecular Targets and Cancer Therapeutics. November 15-19, 2009; Boston, MA; 2009.

101. Huff CA, Padmanabhan S, Kelly KR, et al. A phase I study of an oral hedgehog pathway antagonist, BMS-833923, in patients with relapsed or refractory multiple myeloma. Blood. 2011;118:3993.

102. Jamieson C, Cortes JE, Oehler V, et al. Phase 1 dose-escalation study of PF-04449913, an oral hedgehog ( $\mathrm{Hh})$ inhibitor, in patients with select hematologic malignancies. Blood. 2011;118:424.

103. Yauch RL, Dijkgraaf GJ, Alicke B, et al. Smoothened mutation confers resistance to a Hedgehog pathway inhibitor in medulloblastoma. Science. 2009;326(5952):572-574.

104. Dijkgraaf GJ, Alicke B, Weinmann L, et al. Small molecule inhibition of GDC-0449 refractory smoothened mutants and downstream mechanisms of drug resistance. Cancer Res. 2011;71(2):435-444.

105. Pogoriler J, Millen K, Utset M, Du W. Loss of cyclin D1 impairs cerebellar development and suppresses medulloblastoma formation. Development. 2006;133(19):3929-3937.

106. Lauth M, Bergström A, Shimokawa T, Toftgård R. Inhibition of GLI-mediated transcription and tumor cell growth by small-molecule antagonists. Proc Natl Acad Sci U S A. 2007;104(20):8455-8460.

107. Kim J, Lee JJ, Gardner D, Beachy PA. Arsenic antagonizes the Hedgehog pathway by preventing ciliary accumulation and reducing stability of the Gli2 transcriptional effector. Proc Natl Acad Sci USA. 2010;107(30):13432-13437.

108. Beauchamp EM, Ringer L, Bulut G, et al. Arsenic trioxide inhibits human cancer cell growth and tumor development in mice by blocking Hedgehog/GLI pathway. J Clin Invest. 2011;121(1):148-160.
OncoTargets and Therapy

\section{Publish your work in this journal}

OncoTargets and Therapy is an international, peer-reviewed, open access journal focusing on the pathological basis of all cancers, potential targets for therapy and treatment protocols employed to improve the management of cancer patients. The journal also focuses on the impact of management programs and new therapeutic agents and protocols on

\section{Dovepress}

patient perspectives such as quality of life, adherence and satisfaction The manuscript management system is completely online and includes a very quick and fair peer-review system, which is all easy to use. Visit http://www.dovepress.com/testimonials.php to read real quotes from published authors. 